\title{
Nanoscale Imaging with Force-Based Atomic Force Microscope
}

\section{Joon Won Park}

Dept. of Chemistry, Div. of Integrative Biosci. and Biotech., POSTECH, Republic of Korea

(jwpark@postech.ac.kr)

Second-generation and third-generation dendrons have been applied to AFM probes and substrates to control spacing between the immobilized biomolecules on them. It was observed that use of both probe and substrate allowed one to one interaction during the force measurement. The mapping capability was employed to see mRNAs exposed on sectioned mouse brain tissue, and the enhanced sensitivity was demonstrated. The approach was effective to image a mRNA (AtAMT1;4) on a section of a pollen of Arabidopsis at high resolution. The approach was extended to map individual prostate-specific antibodies captured on a microarrayed spot, and used to calculate the PSA concentration in solution. It was observed that the detection limit was $10 \mathrm{fM}$ concentration, and expected to go down further when a smaller spot and/or scanning larger area were employed. As a new application, picking a biomolecule such as DNA and proteins with the AFM tip was examined. As observed in the force-distance curves, only single copy of the biomolecule was transferred from the substrate to the AFM tip. The capability was applied to pick a single ferritin, a protein having a iron cluster in the core pocket, and the success yield was about 75\%. The AFM probe with a single ferritin at the apex of the tip was used to obtain magnetic force images of magnetic nanoparticles. It was observed that thus-prepared MFM probe was good to image a magnetic particle as small as $10 \mathrm{~nm}$.

\section{References}

1. Yu Jin Jung, Bong Jin Hong, Wenke Zhang, Saul J. B. Tendler, Philip M. Williams, Stephanie Allen,*Joon Won Park,*J. Am. Chem. Soc., 129 (2007) 9349.

2. Jung Sook Kim, Yu Jin Jung, Joon Won Park*, Wei Wang, Wei Wan, and Alexander D. Q. Li*, Adv. Mater., 21 (2009) 786.

3. Yu Jin Jung, Yu Shin Park, Ki-Jun Yoon, Young-Yun Kong, Joon Won Park* and Hong Gil Nam*, Nucleic Acids Research, 37 (2009) e10.

4. Dhruvajyoti Roy, Sung Hong Kwon, Ju-Won Kwak, and Joon Won Park*, Anal. Chem., 82 (2010) 5189.

5. Duckhoe Kim, Nak-Kwan Chung, Stephanie Allen, Saul Tendler, and Joon Won Park*, ACS Nano, 6(2012) 241. 